\title{
Generation of sunspot and polar faculae butterflies using bipolar and quadripolar seed fields
}

\author{
D. K. Callebaut ${ }^{1}$ and A. H. Khater ${ }^{1,2}$ \\ ${ }^{1}$ Physics Department, CGB, University of Antwerp, B-2020 Antwerp, Belgium \\ email: Dirk.Callebaut@ua.ac.be \\ ${ }^{2}$ Department of Mathematics, Faculty of Science, University of Beni Suef, Beni Suef, Egypt \\ email: khater_ah@hotmail.com
}

\begin{abstract}
The exact solution of the evolution equation for the magnetic field in ideal MHD, Callebaut (2006), with an azimuthal velocity which is function of $r$ and $\vartheta$ only (spherical coordinates) is applied to a bipolar magnetic seed field and to a quadripolar field. Resistivity and $\alpha$-effect are not yet taken into account, but the extensions are possible. From the surface observations we had derived an approximate analytic expression for the differential rotation in order to work fully analytically in the application. Qualitatively the results for a quadripolar field are as for a bipolar seed field. The main features are the same: for some latitudes the field may increase by two orders of magnitude, the separation between sunspots and polar faculae is clearcut, there is, relatively speaking, a too strong amplification in the polar regions (the latter occurs in other models too). The hypothesis that the seed fields are situated at the tachocline is not required: the amplification is active throughout the whole convective zone, albeit with different strengths, and thus during the transit of the flux tubes from tachocline to the solar surface too.
\end{abstract}

Keywords. magnetic fields, MHD, dynamo, Sun: sunspots, polar faculae, rotation

\section{Introduction}

When a conducting fluid or a plasma in motion is pervaded by a magnetic field an interaction between the motion and the field takes place and energy may be converted. The field may be increased or decreased by the dynamo action while it looses energy by resistive dissipation. In turn the motion is influenced by the Lorentz force. The applications range from laboratory experiments and industrial use (generation of electricity by dynamos in cars or power plants, pollution problems) to astrophysics. The latter application involves the Sun, stars, galaxies and planets (in particular the Earth). Here we are essentially interested in the solar application, in particular the generation of the butterfly diagrams of the sunspots and of the polar faculae. However, the application has not to be limited to the Sun, in particular as an exact and easy analytical solution is given for the equation of evolution for the magnetic field in the ideal magnetohydrodynamic (MHD) approximation. The basic equations concerned with the magnetic field $\mathbf{H}$ are the equation of evolution for the field together with the conservation of magnetic flux:

$$
\begin{gathered}
\partial_{t} \mathbf{H}=\operatorname{rot}(\mathbf{v} \times \mathbf{H})-\operatorname{rot} \eta \operatorname{rot} \mathbf{H}+\operatorname{rot} \alpha \mathbf{H}, \\
\operatorname{div} \mathbf{H}=0,
\end{gathered}
$$


where $\mathbf{v}$ is the velocity and $\eta=1 / \mu \sigma$ is the resistivity with $\sigma$ the conductivity and $\mu$ the magnetic permeability taken to be constant (SI units).

In general the electromagnetic equations above have to be supplemented by the whole set of (hydrodynamic) equations linked to them through the velocity on the one hand and through the Lorentz force on the other hand. However, in the case of the so-called kinematic dynamo it is assumed that $\mathbf{v}$ is given. This simplification restricts the set of equations to (1) and (2). Moreover, we consider ideal MHD, i.e. $\eta=0$, and we neglect the $\alpha$ term as well for a first approximation.

\section{Solution}

Suppose that the velocity is purely azimuthal and depends only on $r$ and $\vartheta$ :

$$
v_{\varphi}=r \omega(r, \vartheta) \operatorname{sin\vartheta }
$$

where $\omega(r, \vartheta)$ is the angular frequency and $(r, \vartheta, \varphi)$ are spherical coordinates. The general solution of

$$
\partial_{t} \mathbf{H}=\operatorname{rot}(\mathbf{v} \times \mathbf{H})
$$

and equation (1.2), which gives single-valued magnetic field components, is then, Callebaut (2006),

$$
\begin{gathered}
H_{r}=\frac{-\partial_{\vartheta} \Phi}{r^{2} \sin \vartheta}+P_{r}(r, \vartheta, \omega t-\varphi), \\
H_{\vartheta}=\frac{\partial_{r} \Phi}{r \sin \vartheta}+P_{\vartheta}(r, \vartheta, \omega t-\varphi), \\
H_{\varphi}=\frac{-t \partial(\omega, \Phi)}{r \partial(r, \vartheta)}+P_{\varphi}(r, \vartheta, \omega t-\varphi),
\end{gathered}
$$

where the Jacobian is introduced. $P_{r}, P_{\vartheta}$ and $P_{\varphi}$ are purely periodic functions of $\omega t-\varphi$ and contain $r$ and $\vartheta$ in addition. They are completely arbitrary, except that they still have to satisfy equation (2), and, of course, may not have singularities. As we are looking for long-term field amplification and not in the waxing and waning of the field in a timespan of one solar rotation we do not consider them any further here although they may be relevant in other situations.

The stream function $\Phi$ is an arbitrary function of $r$ and $\vartheta$ only. We note that, upon dropping the periodic terms, $H_{r}$ and $H_{\vartheta}$ are independent of time while $H_{\varphi}$ depends linearly on time. Hence there is no violation of the theorem of Cowling which states that no stationary rotationally symmetric dynamo can exist. The linear growth with time is a consequence of the fact that the (differential) rotation is stationary.

\section{Choice of the seed field}

Equations (2.3-5) give the exact solution of the equation of evolution in ideal MHD. Given the value of $\mathbf{H}$ at any particular time we can write down immediately the magnetic field at all times. For the desired field amplification in the Sun we have thus to find a seed magnetic field. As we are not concerned at the moment with the periodic terms in equations (2.3-5), the choice is already somewhat limited: a seed field independent of $\varphi$ and in which the $H_{r}$ and $H_{\vartheta}$ components are linked by the flux conservation as in equation (1.2). This limits the freedom to one component dependent on $r$ and $\vartheta$ only. We first consider the localization of the seed field. Broadly speaking the following localizations 
are possible: the radiative core, the convective zone and the tachocline, separation and link between the previous ones.

\subsection{Fossil field in the core}

In view of the gigantic dimensions of the Sun (and its core) and thus the very slow resistive dissipation a fossil field may be located there. Considering in equation (1.1) on the right hand side the resistive term only and supposing a periodic field in space, with wavelength $\lambda$, yields for the characteristic decay time

$$
\tau=\frac{\lambda^{2}}{4 \pi^{2} \eta} .
$$

The largest $\lambda$ to be expected should correspond to half a wavelength equal to the diameter of the core, i.e. $\lambda / 2=2 r_{0}$. For $r_{0}=510^{8} \mathrm{~m}$ and taking $\eta=1 \mathrm{~m}^{2} / \mathrm{s}$, as the matter is fully ionized (copper has slightly more than $\eta=0.01 \mathrm{~m}^{2} / \mathrm{s}$ ) we obtain $\tau=10^{17} \mathrm{~s}$ or $310^{9}$ year. Hence a fossil field is quite possible, as the lifetime of the Sun is not even twice this value. However, the wavelengths that are much smaller, e.g. 10 times smaller, will have a characteristic time which is 100 times smaller and hence they may have practically vanished. This is important when considering multipolar fields. On the other hand there may still be a bit of differential rotation in the core, which after say some thousand years may have resulted in a fairly strong field, which moreover may have moved radially upward by the buoyancy force to the tachocline, Dudorov, Krivodubskij, Ruzmaikina \& Ruzmaikin(1989).

Hence we get the following picture: at the basis of the tachocline we get a fair field, whether this is provided by the fossil field alone or in combination with the small differential rotation in the core. This field is further amplified strongly by the differential rotation in the tachocline itself resulting in a very strong field when it has reached (after many years, say several solar cycles) the upper side of the tachocline, Krivodubskij (2005). In fact the observations suggest a very strong field in the tachocline: of the order of 100 kgauss. However, this field may be in layers or filaments. When sufficiently strong these peel off the tachocline and evolve further toward the solar surface, while the field may be further amplified by the differential rotation in the convective zone. Taking a thickness of such a layer as $100 \mathrm{~km}(\approx \lambda / 2)$ yields $10^{9} \mathrm{~s}$ or 30 years as a characteristic decay time (see expression (3.1)). This means a fairly fast resistive decline for the field and the amplification should be very strong to compensate for it. Thinner layers are practically excluded. If one layer peels off in one cycle and the tachocline is about $10000 \mathrm{~km}$, then the latter may provide about 100 cycles, or a 1000 years. However, the tachocline is continually renewed from below. Clearly subsequent layers should have fields strengths of opposite signs to account for the Hale cycle. Actually this creates a paradox with the presumed large wavelengths considered above to keep a remnant of the fossil field. However, the strong differential rotation, the overshooting of the radiative zone and the turbulence in the tachocline may be responsible for this thin filamentation in the tachocline.

\subsection{Regeneration of global magnetic surface fields}

The large-scale unipolar magnetic field regions at the solar surface (in short: global field regions) are generated near the solar equator. Their number varies from 4 to 8 . Neighboring regions have alternating signs: if the field dives into the Sun in one, then it goes out of the Sun in the neighboring ones. Their boundaries correspond to filaments bands (filaments and filament channels). These global regions move toward the poles except during some time in the minimum of the solar activity, when they oscillate around their so-called 'rest-latitudes', Callebaut \& Makarov (1992), Makarov, Tlatov, Callebaut 
et al. (2002), Makarov, Makarova \& Callebaut (2006). What happens at the poles is not perfectly clear: part of the energy has been dissipated and part of it dives into the Sun. The latter part may be brought back to the equatorial region, by the meridional motion and reappear at the solar surface. This corresponds to the so-called flux-transport dynamo. A good argument for this was given by Charbonneau \& Dikpati (2000) who showed through numerical simulation of their flux-transport dynamo that the shear-layer toroidal fields of the cycle $n$ have the strongest positive correlation with the polar fields of the cycle $n-2$ compared to that of the cycles $n, n-1$ and $n-3$. They state that this correlation is the consequence of the 17-21 years duration of the Sun's memory about its past magnetic fields. We refer to Dikpati, de Toma, Gilman, et al. (2004), Dikpati (2005) and Dikpati (2006).

There is another link which may be clarifying for this flux-transport dynamo theory. It was shown by Makarov \& Makarova (1996) that the peaks in the number of polar faculae are correlated with the peaks in sunspot number. Makarov succeeded in predicting from these data that the maximum Wolf number in cycle 23 would be at most 130 , and indeed it came out to be about 120 . The relation between polar faculae was further clarified by Makarov, Makarova \& Callebaut (2006): the peaks correspond, but the total number of polar faculae and the total sunspot number do not show an obvious correlation. This suggests that part of these phenomena are correlated either through the flux-transport dynamo and the meridional motion or the $\alpha$-effect while another part is generated elsewhere, that is in the tachocline region. Clearly the solution for the amplification of the magnetic field given in section 2 applies to both situations. However, as we expect that the contribution coming from the tachocline is the larger one we shall elaborate this case.

\subsection{Expression for a bipolar field}

Any magnetic field located in the central part of the Sun may be approximated by a multipolar field. The Earth and several planets have to a good approximation a dipolar field and thus it is indicated to start with a dipolar field as a first trial. Moreover, as the Sun has a fairly symmetrical behavior when averaging over several cycles, we take the axis along the axis of rotation. Hence we consider the following bipolar field as seed field:

$$
H_{b r}=\frac{H_{b} \cos \vartheta}{r^{3}}, \quad H_{b \vartheta}=\frac{H_{b} \sin \vartheta}{2 r^{3}},
$$

where $H_{b}$ is a constant and where the index $b$ is used to distinguish from the quadripolar field with index $q$ in next subsection. The field may be situated at the tachocline or any place in the convective zone. The third component $H_{b \varphi}$ may have any initial value independent of $\varphi$; it will further take its appropriate value according to the solution of the evolution equation given above. As we are mainly interested in the growth of the field we omit the time independent part of $H_{b \varphi}$. From equation (3.2) we deduce

$$
\Phi=\frac{-H_{b} \sin ^{2} \vartheta}{2 r}, \quad \partial_{r} \Phi=\frac{H_{b} \sin ^{2} \vartheta}{2 r^{2}}, \quad \partial_{\vartheta} \Phi=\frac{-H_{b} \sin \vartheta \cos \vartheta}{r}
$$

\subsection{Expression for a quadripolar field}

The bipolar field is certainly a good start. However, the tachocline is situated around $0.7 R$ and at this (not quite large) distance from the solar center the influence of higher order polarities will not be negligible. Hence, as a second example, we consider the following quadripolar field as seed field:

$$
H_{q r}=\frac{H_{q} \cos \vartheta}{r^{4}}, \quad H_{q \vartheta}=\frac{H_{q} \sin \vartheta}{r^{4}}
$$


while the time independent part of $H_{q \varphi}$ is omitted. $H_{q}$ is again a constant. When a combination of a bipolar and a quadripolar one is used the ratio $r H_{b} / H_{q}$ will be relevant. We attempted to estimate this value from the observations in Makarov, Tlatov, Callebaut et al. (2001), but this did not yet work out. In agreement with (3.4) we have for the corresponding stream function $\Psi$ :

$$
\Psi=\frac{-H_{q} \sin ^{2} \vartheta}{2 r^{2}}, \quad \partial_{r} \Psi=\frac{H_{q} \sin ^{2} \vartheta}{r^{3}}, \quad \partial_{\vartheta} \Psi=\frac{-H_{q} \sin \vartheta \cos \vartheta}{r^{2}} .
$$

The difference with the bipolar field is not strong: the power of $r$ in the denominator is a unit more in all expressions here and a factor 2 occurs in some terms; thus it is mainly the $r$-dependence which will be investigated by comparison with the bipolar case. It is possible to investigate the $\vartheta$-dependence by using $\cos ^{n} \vartheta \sin ^{m} \vartheta$ with $n=0,1,2,3, \ldots$ and $m=2,3, \ldots$ in he numerator of $\Phi$ and $\Psi$.

\section{Analytic expression for the differential rotation}

Helio-seismology provides us with data for the differential rotation, Kosovichev, Schou, Scherrer et al. (1997). As we have an analytic expression for the magnetic field we approximate the differential rotation by an analytic expression, using some simplifying suppositions: the radial dependence of $\omega$ varies linearly with $r$ and we take $\vartheta_{0}$ where $\partial_{r} \omega=0$, corresponding to the conical blades in the convective zone where the angular frequency remains constant $\left(\vartheta_{0} \approx 53^{\circ}\right)$. We obtained

$$
\omega=\omega_{r_{0}}+\frac{a\left(r-r_{0}\right)\left(\cos ^{2} \vartheta_{0}-\cos ^{2} \vartheta\right)\left(1+b \cos ^{2} \vartheta\right)}{\left(R-r_{0}\right) \cos ^{2} \vartheta_{0}} .
$$

Here $r_{0}$ is the radius of the tachocline. We shall take $r_{0}=0.7 R$, with $R$ the solar radius. Moreover we have approximately $a=5.77$ and $b=.87$.

\section{Numerical results}

\subsection{The seed field is a bipolar one}

Combining the relevant equations for $\Phi$ and $\omega$ given in sections (3.3) and (4) yields $H_{b \varphi}$ :

$$
\begin{aligned}
H_{b \varphi}= & \frac{5.77 H_{b} \operatorname{tsin} \vartheta \cos \vartheta}{r^{3}\left(R-r_{0}\right) \cos ^{2} \vartheta_{0}}\left[r\left(\cos ^{2} \vartheta_{0}-\cos ^{2} \vartheta\right)\left(1+0.87 \cos ^{2} \vartheta\right)\right. \\
& \left.+\left(r-r_{0}\right) \sin ^{2} \vartheta\left(1-0.87 \cos ^{2} \vartheta_{0}+1.74 \cos ^{2} \vartheta\right)\right] .
\end{aligned}
$$

We have tabulated the results for $\cos \vartheta_{0}=0.6$ and $r_{0}=0.7 R$ in table 1 . We took $r=0.7 R$, respectively $r=R$ to have an idea how the amplification is changing across the convective zone, i.e. how the amplification is changing during the travel of the flux tube through the convective zone. Plotted are $R^{3} H_{b \varphi} / t H_{b}$ and the multiplication factor per year $X=r^{3} H_{b \varphi} / H_{b} \sqrt{\cos ^{2} \vartheta+0.25 \sin ^{2} \vartheta}$ which is the ratio of $H_{b \varphi}$ after one year and $\sqrt{H_{b r}^{2}+H_{b \varphi}^{2}}$.

The general trend is qualitatively the same for both values of $r$ : no growth at the equator $\left(\vartheta=90^{\circ}\right)$, a fair growth at latitudes $10^{\circ}$, the largest growth at $20^{\circ}$ and further a decrease to very slow growth (or even reversing growth) near $\vartheta_{0}$. For the sunspot diagram this seems satisfactory for a first attempt. For the higher latitudes one finds again a strong growth (constituting a problem: see below) turning to zero growth at the poles. However quantitatively there is quite a difference between $r=0.7 R$ and $r=R$; moreover their ratios are inverted when passing from the sunspot zone to the polar faculae zone. 


\begin{tabular}{c|c|c|c|c}
$\vartheta$ & $r=R \mid X(R)$ & \multicolumn{2}{c}{$r=0.7 R$} & $X(0.7 R)$ \\
\hline $90^{\circ}$ (eq.) & 0 & 0 & 0 & 0 \\
$80^{\circ}$ & 5.04 & 9.7 & 6.30 & 4.1 \\
$70^{\circ}$ & 8.63 & 14.9 & 9.37 & 5.5 \\
$60^{\circ}$ & 8.93 & 13.5 & 6.32 & 3.3 \\
$50^{\circ}$ & 4.60 & 6.1 & -3.81 & -1.7 \\
$40^{\circ}$ & -1.15 & -1.4 & -18.3 & -7.6 \\
$30^{\circ}$ & -2.97 & -3.3 & -30.3 & -11.6 \\
$20^{\circ}$ & -3.75 & -3.9 & -32.2 & -11.7 \\
$10^{\circ}$ & -1.56 & -1.6 & -20.9 & -7.2 \\
$0^{\circ}$ & 0 & 0 & 0 & 0
\end{tabular}

Table 1. Growth of $H_{b \varphi}$ and of the amplification factor per year $X$ (from equator to pole) for $r_{0}=0.7 R$.

As $\omega$ is expressed per year we have $t$ in the expression for $H_{\varphi}$ in years too. This means that with a yearly increase by a factor 10 we obtain a multiplication by about 100 in one cycle at latitudes of $20^{\circ}$ near the surface. As the seed value of the magnetic field may be easily 1 to 100 kgauss the result is fair (due to the expansion of the flux tubes when rising toward the solar surface an upper limit of about 5000 gauss arises). Indeed, the growing magnetic flux tube will evolve to larger $r$ due to the buoyancy force and/or convection, but the growth process continues more or less at the same trend for all latitudes except around $\vartheta_{0}$. That the sunspots near $10^{\circ}-20^{\circ}$ appear later than those at $30^{\circ}$ may be due to a hampering effect of the centrifugal force close to the equator. Indeed, around the equatorial plane the angular frequency increases, which counteracts the buoyancy force when magnetic flux tubes with plasma are rising toward the surface. The fact that larger sunspots appear during the second half of a cycle and precisely in the latitude region from $15^{\circ}-25^{\circ}$ may be seen as a confirmation of this effect: the field needed a longer time to overcome the adverse angular frequency gradient and thus grew stronger, resulting in larger sunspots.

Clearly $\vartheta_{0}$, marking the region where $\partial_{r} \omega=0$, corresponds to a separation between two regions with different character, the sunspot region and the polar faculae region. It may be noted that in the region around $\vartheta_{0}$ the field may grow for lower values of $r$, while close to the surface it grows in the opposite sense, thus (when the flux tube is rising to the surface) diminishing the first increase, clearly resulting in poor magnetic activity. This gives a clear division in latitude between the polar faculae region and the sunspot region, although basically they are generated by the same mechanism.

Remark that $H_{\varphi}$ is proportional to $\sin \vartheta$, thus yielding results of the opposite sign in the hemispheres, as it should be in view of the opposite order of polarity between the leading $\operatorname{spot}(\mathrm{s})$ and the follower(s) in both hemispheres. We made a calculation for $r_{0}=0.5 R$ too (exaggerating strongly the possible variation on the location of the tachocline): this yields in general a similar but smaller amplification. Note that for high latitudes and close to the tachocline $\left(r_{0}=0.7 R\right)$ the growth may be quite fast too. Although the approximation for $\omega$ may be rather poor for the high latitudes this trend may be partially real. In fact at the higher latitudes the angular frequency is decreasing with distance from the center of the Sun. Thus the centrifugal force is helping the buoyancy force there. It follows that the rising magnetic flux and plasma tubes pop up rather easily and quickly, resulting in less time to grow, thus resulting in weaker fields and smaller areas (polar faculae, with fields up to 1.5 kgauss) than in the equatorial region (sunspots). Moreover, as the time spend by a rising flux and plasma tube at the high latitudes is presumably short near the solar surface the large growth rates near $r=R$ have there little effect on the magnetic phenomena coming from deeper regions. However, these growth rates near the surface 
may be important for some phenomena, like the regeneration of the global magnetic field, which is maybe located in the body of the convective zone and not at its bottom.

The helping hand of the centrifugal force to the buoyancy force at high latitudes may explain too that the polar faculae cycle preceeds the sunspot cycle by about 5 years as clearly shown in several papers (Makarov, Makarova \& Sivaraman (1989), Makarov \& Makarova (1996), Makarov, Tlatov \& Sivaraman (2003), Makarov, Tlatov \& Callebaut (2004), Makarov, Makarova \& Callebaut (2006)). Makarov now uses the polar phenomena with success to predict the behavior of the next sunspot cycle (maximum Wolf number and peaks in the monthly Wolf numbers). Moreover, there are arguments that near the bottom of the convective zone in the equatorial region there is an additional push toward the solar surface as a consequence of the rotation, Krivodubskij (1992), while the same effect is retarding the upward motion of the flux tubes in all other parts of the convective zone. Nevertheless a delay of half a solar cycle seems a lot so the action of centrifugal force (delaying the rising of the flux tubes in the sunspot region, speeding them up in the polar region) may not be sufficient to explain about half a cycle between the peaks in the number of polar faculae and the peaks in sunspot number. A neat calculation of the centrifugal force versus the buoyancy force based on the gradients of the density and rotational velocity is required here.

\subsection{The seed field is a quadripolar one}

Combining the relevant equations for $\Psi$ and $\omega$ given in sections (3.4) and (4) yields $H_{q \varphi}$ :

$$
\begin{aligned}
H_{q \varphi}= & \frac{5.77 H_{q} t \sin \vartheta \cos \vartheta}{r^{4}\left(R-r_{0}\right) \cos ^{2} \vartheta_{0}}\left[r\left(\cos ^{2} \vartheta_{0}-\cos ^{2} \vartheta\right)\left(1+0.87 \cos ^{2} \vartheta\right)\right. \\
& \left.+2\left(r-r_{0}\right) \sin ^{2} \vartheta\left(1-0.87 \cos ^{2} \vartheta_{0}+1.74 \cos ^{2} \vartheta\right)\right]
\end{aligned}
$$

Again the change with a bipolar field looks small: $r^{3}$ is replaced by $r^{4}$ and a factor 2 appears in the last term. We have tabulated the results again for $\cos \vartheta_{0}=0.6$ and $r_{0}=0.7 R$ in table 2 . We took again $r=0.7 R$, respectively $r=R$. Plotted are now $R^{4} H_{q \varphi} / t H_{q}$ and the multiplication factor per year $Y=r^{4} H_{q \varphi} / H_{b}$ which is the ratio of $H_{q \varphi}$ after one year and $\sqrt{H_{q r}^{2}+H_{q \varphi}^{2}}$.

\begin{tabular}{c|c|c|c|c}
$\vartheta$ & $r=R \mid Y(R)$ & \multicolumn{2}{c}{$r=0.7 R$} & $Y(0.7 R)$ \\
\hline $90^{\circ}$ (eq.) & 0 & 0 & 0 & 0 \\
$80^{\circ}$ & 8.05 & 8 & 9.01 & 2.2 \\
$70^{\circ}$ & 12.7 & 13 & 13.5 & 3.2 \\
$60^{\circ}$ & 14.7 & 15 & 9.24 & 2.2 \\
$50^{\circ}$ & 11.1 & 11 & -5.53 & -1.3 \\
$40^{\circ}$ & 2.12 & 2.1 & -26.3 & -6.3 \\
$30^{\circ}$ & -7.8 & -7.8 & -43.5 & -10 \\
$20^{\circ}$ & -13.2 & -13 & -46.3 & -11 \\
$10^{\circ}$ & -9.87 & -9.9 & -29.9 & -7.2 \\
$0^{\circ}$ & 0 & 0 & 0 & 0
\end{tabular}

Table 2. Growth of $H_{q \varphi}$ and of the amplification factor per year $Y$ (from equator to pole) for $r_{0}=0.7 R$.

Comparison of both tables shows that qualitatively the results are very similar. The amplification in the polar regions is even more accentuated for the quadripolar field than for the bipolar one. This has clearly to be ascribed to the strong differential rotation near the poles. This problem is encountered by several models, Ossendrijver (2003), Dikpati (2005). 


\section{Conclusions}

We have used the analytic solution of the equation of evolution for the magnetic field in ideal MHD to calculate the field amplification in the convective zone. We used an analytic expression for the angular frequency of the differential rotation. We considered once a bipolar and once a quadripolar seed field and found fair amplification which may amount to two orders of magnitude in one cycle in the sunspot region and at high latitudes; that the amplification is apparently too strong at those high latitudes is a drawback occurring in other models too. We ascribe it, at least partially, to the delaying action of the centrifugal force in the sunspot region, counteracting the buoyancy force, that the sunspots grow larger than the polar faculae. There is a clear cut between the butterfly diagrams of the sunspots and polar faculae, although basically the mechanism is the same for both. The theory allows expansion to include flux-transport from the global magnetic field regions, Callebaut, Hady, Karugila \& Khater (2006), and the $\alpha$-effect as well.

\section{Acknowledgements}

It is a pleasure to thank Prof. Em. Dr. C. de Jager (formerly at the University of Utrecht, Utrecht, the Netherlands) for many delightful discussions.

\section{References}

Callebaut, D.K. \& Makarov, V.M. 1992, Solar Phys, 141, 381

Callebaut, D.K. 2006, ApJ, submitted

Callebaut, D.K., Makarov, V.I. \& Tlatov, A.G. 2002 in: Proc. 10th Eur. Solar Phys. Meeting, Prague, Czech Republic, 9-14 Sept 2002 'Solar variability from core to outer frontiers' ESA SP-506, Dec., 113

Callebaut, D.K., Hady, A.A., Karugila, G.K. \& Khater, A.H. 2006, IAUS233, these proceedings

Charbonneau, P. \& Dikpati, M. 2000, ApJ 543, 1027

Dikpati, M., de Toma, G., Gilman, P.A., Arge, C.N. \& White,O.R. 2004 ApJ 601, 1138

Dikpati, M. 2005 Adv. Space Res. 35, 322

Dikpati, M. 2006 Geoph. Res. L 33, L05102

Dudorov, A.E., Krivodubskij, V.N., Ruzmaikina, T.V. \& Ruzmaikin, A.A. 1989 Sov.Astron 33 (4), 420

Kosovichev, A.G., Schou, J., Scherrer, P.H. \& 31 co-authors 1997 Solar Phys 170, 43

Krivodubskij, V.N. 1992 Sov.Astron 36 (4), 432

Krivodubskij, V.N. 2005 Astron. Nachr. 326 (1), 61

Makarov, V.I. \& Makarova, V.V. 1996 Solar Phys. 163, 267

Makarov, V.I, Tlatov, A.G., Callebaut, D.K., Obridko, V.N. \& Shelting, B.D. 2001 Solar Phys. 198, 409

Makarov, V.I, Tlatov, A.G., Callebaut, D.K. \& Obridko, V.N. 2002 Solar Phys. 206, 383

Makarov, V.I., Tlatov, A.G. \& Sivaraman, K.R. 2003 Solar Phys. 214, 41

Makarov, V.I., Tlatov, A.G. \& Callebaut, D.K. 2004 Solar Phys. 224, 49

Makarov, V.I, Makarova, V.V. \& Callebaut, D.K. 2006 Solar Phys. in press

Ossendrijver, M. 2002 in: Proc. 10th Eur. Solar Phys. Meeting, Prague, Czech Republic, 9-14

Sept 2002 'Solar variability from core to outer frontiers' ESA SP-506, Dec., 797

Ossendrijver, M., Stix, M., Brandenburg, A., \& Rüdiger, G. 2002 A\& A 394, 735

Ossendrijver, M. 2003 A $\& A$ Rev 11, 287

Wang, Y.-M. \& Sheeley jr, N. R. 1991, ApJ 375, 761 